\title{
Whole-brain ex-vivo quantitative MRI of the cuprizone mouse model
}

\author{
Tobias C Wood ${ }^{\text {Corresp.. }}{ }^{1}$ ， Camilla Simmons ${ }^{1}$ ， Samuel A Hurley ${ }^{2,3}$ ， Anthony C Vernon ${ }^{4}$, Joel Torres ${ }^{1}$, Flavio \\ Dell'Acqua $^{1,5}$, Steve C R Williams ${ }^{1}$, Diana Cash ${ }^{1}$ \\ 1 Department of Neuroimaging, IOPPN, King's College London, London, United Kingdom \\ 2 FMRIB Centre, Nuffield Department of Clinical Neurosciences, University of Oxford, Oxford, Oxfordshire, United Kingdom \\ 3 Synaptive Medical, Toronto, ON, Canada \\ 4 Cells and Behaviour Unit, Department of Basic and Clinical Neuroscience, IOPPN, King's College London, London, United Kingdom \\ 5 NatBrainLab, Department of Basic and Clinical Neuroscience, IOPPN, King's College London, London, United Kingdom \\ Corresponding Author: Tobias C Wood \\ Email address: tobias.wood@kcl.ac.uk
}

Myelin is a critical component of the nervous system and a major contributor to contrast in Magnetic Resonance (MR) images. However the precise contribution of myelination to multiple MR modalities is still under debate. The cuprizone mouse is a well established model of demyelination that has been used in several MR studies, but these have often imaged only a single slice and analysed a small region of interest in the corpus callosum. We imaged and analyzed the whole brain of the cuprizone mouse ex-vivo using highresolution quantitative MR methods (multi-component relaxometry, Diffusion Tensor Imaging and morphometry) and found changes in multiple regions, including the corpus callosum, cerebellum, thalamus and hippocampus. However the presence of inflammation, confirmed with histology, presents difficulties in isolating the sensitivity and specificity of these MR methods to demyelination using this model. 


\title{
Whole-Brain Ex-Vivo Quantitative MRI of the Cuprizone Mouse Model
}

\author{
Tobias C Wood ${ }^{1}$, Camilla Simmons ${ }^{1}$, Samuel A Hurley ${ }^{2,3}$, Anthony \\ Vernon $^{4}$, Joel Torres ${ }^{1}$, Flavio Dell'Acqua ${ }^{1,5}$, Steve C R Williams ${ }^{1}$, and \\ Diana Cash ${ }^{1}$ \\ ${ }^{1}$ Department of Neuroimaging, IOPPN, King's College London \\ ${ }^{2}$ FMRIB Centre, Nuffield Department of Clinical Neurosciences, University of Oxford \\ ${ }^{3}$ Synaptive Medical, Toronto, Canada \\ ${ }^{4}$ Cells and Behaviour Unit, Department Basic and Clinical Neuroscience, IOPPN, King's \\ College London \\ ${ }^{5}$ NatBrainLab, Department of Forensic and Neurodevelopmental Sciences, IOPPN, \\ King's College London
}

\begin{abstract}
Myelin is a critical component of the nervous system and a major contributor to contrast in Magnetic Resonance (MR) images. However the precise contribution of myelination to multiple MR modalities is still under debate. The cuprizone mouse is a well established model of demyelination that has been used in several MR studies, but these have often imaged only a single slice and analysed a small region of interest in the corpus callosum. We imaged and analyzed the whole brain of the cuprizone mouse ex-vivo using high-resolution quantitative MR methods (multi-component relaxometry, Diffusion Tensor Imaging and morphometry) and found changes in multiple regions, including the corpus callosum, cerebellum, thalamus and hippocampus. However the presence of inflammation, confirmed with histology, presents difficulties in isolating the sensitivity and specificity of these MR methods to demyelination using this model.
\end{abstract}

Keywords: cuprizone, MRI, myelin, quantitative imaging

\section{INTRODUCTION}

Myelin is a critical component of a healthy nervous system. It is composed of protein and lipid layers that tightly wrap neurons, improving their electrical conductivity and reducing their energy requirements (Nave and Werner, 2014). Focal demyelinating lesions are the hallmark of Multiple Sclerosis and disruption of myelin is also associated with other neurodegenerative diseases such as Alzheimer's and Parkinson's Disease. Non-invasive methods to quantify the myelination state of the nervous system are hence highly useful in order to better track the progression of these diseases, and any protective or regenerative treatments that become available (Dubessy et al., 2014).

Myelin is also a uniquely useful structure for MRI as it contributes to almost every known contrast mechanism. The high lipid content provides abundant pathways for spin-lattice interactions, reducing the longitudinal relaxation time (T1) of surrounding protons (Stuber et al., 2014) and generating significant Magnetization Transfer (MT) effects (Turati et al., 2014). It is impermeable, hindering the diffusion of water molecules (Song et al., 2005). Myelin is diamagnetic compared to grey matter (GM), leading to excellent phase and susceptibility contrast (Lee et al., 2012). The unique layered structure traps water protons in restricted environments, leading to reduced transverse relaxation (T2) and an additional inhomogeneous or dipolar MT effect (Mackay et al., 1994; Varma et al., 2015). It is hence of little surprise to find great interest in quantifying myelin content using MR techniques.

Although numerous approaches have been proposed to quantify myelin with MRI, proper validation of such methods can be lacking in the literature. A particular case in point is the multi-component Driven-Equilibrium Single-Pulse Observation of T1/T2 (mcDESPOT) method. This aims to divide the MR signal resulting from steady-state sequences into three pools representing water trapped in the 


\begin{tabular}{|l|c|c|c|c|}
\hline Citation & $\begin{array}{c}\text { In-/ } \\
\text { Ex-Vivo }\end{array}$ & $\begin{array}{c}\text { Acquired } \\
\text { Volume }\end{array}$ & $\begin{array}{c}\text { Analysis } \\
\text { Volume }\end{array}$ & Methods \\
\hline Song et al. (2005) & Ex & MS & CC, EC, OT, CP & DTI \\
Merkler et al. (2005) & In & 3D & CC, EC & T1w, T2w, MTR \\
Sun et al. (2006) & In & MS & CC & DTI \\
Wu et al. (2008) & In & MS & CC & T2w, DTI \\
Torkildsen et al. (2009) & In & MS & Lesion volumes & T2w \\
Xie et al. (2010) & In & MS & CC & DTI \\
Zhang et al. (2012) & Both & 3D/MS & CC, Ctx & T2w, MTR, DTI \\
Chandran et al. (2012) & In & MS & CC, Cg, EC & T2w, DTI \\
Thiessen et al. (2013) & Both & SS & CC, EC, Ctx & T1/2, qMT, DTI, MWF \\
Fjær et al. (2013) & In & 3D & CC, GM, Cbl, Ctx & T2w, MTR \\
Falangola et al. (2014) & In & MS & CC & DKI \\
Turati et al. (2014) & In & SS $\times 2$ & CC, EC & qMT \\
Guglielmetti et al. (2016) & In & MS & CC, Ctx & T2w, DKI \\
Jelescu et al. (2016) & In & SS & CC & T2w, MTR, DKI \\
Tagge et al. (2016) & In & 3D & Cbr & T2w, MTR \\
\hline
\end{tabular}

Table 1. A review of existing cuprizone literature, showing the wide variety of MR modalities used. A majority of papers analyzed a small ROI in the Corpus Callosum. Abbreviations: SS - Single-slice, MS Multi-slice, CC - Corpus Callosum, EC - External Capsule, Ctx - Cortex, Cg - Cingulum, Cbl Cerebellum, Cbr - Cerebrum, OT - Optic Tracts, CP - Cerebral Peduncles, T1w - $T_{1}$ Weighted, T2w $-T_{2}$ Weighted, T1 - T1 Map, T2 - T2 Map, MTR - Magnetisation Transfer Ratio, qMT - Quantitative Magnetisation Transfer, DTI - Diffusion Tensor Imaging, DKI - Diffusion Kurtosis Imaging, MWF Myelin Water Fraction.

myelin sheath, intra-extracellular water, and free water in cerebrospinal fluid (Deoni et al., 2013). It has been used in several clinical studies, however to our knowledge it has had little validation with pre-clinical or histological studies. It has also been criticized for theoretical difficulties with the fitting procedure (Lankford and Does, 2012; Zhang et al., 2014), but these have been partially addressed by subsequent literature (Hurley and Alexander, 2014; Bouhrara and Spencer, 2015). In this paper we provide a practical demonstration of mcDESPOT in the widely-used cuprizone mouse model of demyelination (Torkildsen et al., 2008; Skripuletz et al., 2011). In this model mice are fed cuprizone over a period of weeks, and progressive demyelination can be observed throughout white matter (Goldberg et al., 2015).

The cuprizone model has already been extensively imaged with multiple MR techniques, which are summarized in table 1. However, the majority of these studies were restricted to imaging just one or two slices of the brain, often chosen to cover sections of the corpus callosum. This negates one of the major benefits of MRI, which is the ability to image the entire brain in a reasonable amount of time. The analyses are then often conducted on a small number of Regions-Of-Interest (ROIs), reducing the rich information available in MRI to a single composite number.

Hence a further aim of this study was to acquire full-brain, high resolution MRI of the cuprizone model, and look for effects outside of the corpus callosum. Such methodology is widespread in clinical MR studies, so their use in pre-clinical studies will aid translatability. We are aware of only two cuprizone papers that attempted similar acquisitions, however Tagge et al. (2016) did not achieve sufficient SNR in the cerebellum and Fjær et al. (2013) did not conduct histology there.

The comprehensive study of Thiessen et al. (2013) attempted to measure the MWF with the quantitative Multi-Echo T2 (MET2) method but failed to find a MWF even in healthy white matter (WM) at 7T. MET2 is considered somewhat of a gold standard for MWF imaging, so this study was an opportunity to evaluate whether mcDESPOT could robustly detect a MWF. Using ex-vivo imaging we could acquire a greater number of MR modalities at higher resolution than would be feasible in-vivo. In addition to the structural and relaxometry data, we also acquired Diffusion Tensor Imaging (DTI) data, allowing direct comparison of the sensitivity and specificity of DTI to relaxometry across the whole brain.

To summarise, the aims of this experiment were: 
1. Demonstrate the feasibility of MWF imaging using the mcDESPOT technique in a pre-clinical model.

2. Observe the effects of cuprizone treatment across the entire encephalon using multiple quantitative MR methods.

3. Use histological validation to assess their sensitivity to demyelination and inflammation.

\section{METHODS}

\section{Animal Model}

All experiments were performed under approval of the local King's College London ethics committee and the UK Animals (Scientific Procedures) Act 1986, according to the Home Office Project License Number $70 / 8480$ (held by Diana Cash). For a thorough description of the cuprizone model the reader is referred to Skripuletz et al. (2011). Seven adult male C57BL/6J (Harlan, UK) mice were housed communally and freely fed powdered rodent chow for five weeks, while a further eight were fed powdered chow mixed with $0.2 \%$ cuprizone (Bis(cyclohexanone)oxaldihydrazone, Sigma Aldrich UK). All animals were weighed weekly. The five week time-point was chosen as heavy demyelination is expected to be present. They were then killed by transcardiac perfusion with ice-cold heparinized saline followed by $4 \%$ buffered paraformaldehyde (PFA). The heads were removed, stored in PFA for 24 hours and then rehydrated in Phosphate Buffered Saline preserved with $0.05 \%$ sodium azide at $4^{\circ}$ for a minimum of 30 days (Cahill et al., 2012).

After image acquisition one control and one cuprizone mouse were excluded from the MRI analysis as the cerebellum had been damaged during sample preparation, preventing a good registration in this region, however the rest of the brain could be used for histology. For quantitative histology, high quality sections could be obtained from all cuprizone animals but only five controls.

\section{MRI Acquisition}

MR Images were acquired using a 7 Tesla pre-clinical MR system (Agilent Technologies). Samples were immersed in fluorinated liquid to reduce susceptibility artefacts (Galden, Solvay) and loaded four at a time (see figure 1 into a 39mm diameter transmit-receive birdcage coil (Rapid GmbH). Three sets of MR images were acquired; a 3D Fast-Spin Echo for structural analysis, a mcDESPOT protocol, and a DTI protocol.

The 3D FSE image had a matrix size of 256x256x256 with isotropic $112.5 \mu \mathrm{m}$ voxels, TE/TR = $40 / 3000 \mathrm{~ms}$, an echo-train length of 16 , echo-spacing $6.67 \mathrm{~ms}$, readout bandwidth $62.5 \mathrm{kHz}$, and scan time 3 hours 25 minutes. The DTI scans were acquired using a four shot EPI sequence with a $192 \times 128$ matrix, 40 slices, $150 \times 225 \times 500 \mu \mathrm{m}$ voxel size, TE/TR=43/4000ms, 10 averages and scan time was 3 hours 7 minutes. The diffusion preparation consisted of 30 diffusion directions with $b=2000$ and four $b=0$ images, with $\delta / \Delta=4 / 16 \mathrm{~ms}$.

The mcDESPOT protocol consisted of a SPoiled Gradient Recalled (SPGR) scan, a balanced SteadyState Free-Precession (bSSFP) scan, and an Actual Flip-angle Imaging (AFI) scan for B1 inhomogeneity

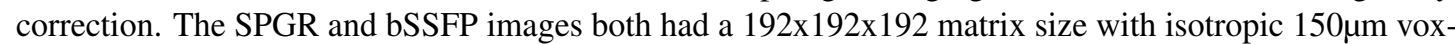
els. The SPGR images had TE/TR=5.148/20ms, with a read-out bandwidth of $25 \mathrm{kHz}$, and were acquired at 12 flip-angles $\left(4,5,6,8,10,12,16,20,24,26,28,30^{\circ}\right)$, scan time 2 hours 28 minutes, and a strong diffusion spoiling scheme. The bSSFP images were acquired with TE/TR $=3 / 6 \mathrm{~ms}$, a band-width of $62.5 \mathrm{kHz}$, also 12 flip-angles $\left(8,9,10,12,15,20,30,40,50,55,60,65^{\circ}\right)$ and scan time 2 hours 57 minutes. The extreme flipangles were chosen to be the optimal for the expected single-component T1 and T2 values (Wood, 2015). The AFI scan had a matrix size of $96 \times 96 \times 96$, isotropic $300 \mu \mathrm{m}$ voxels, TE/TR1/TR2 $=4.304 / 20 / 100 \mathrm{~ms}$, bandwidth $15.625 \mathrm{kHz}$, a flip-angle of $55^{\circ}$ and scan time 37 minutes (Yarnykh, 2007, 2010). The acquisition protocol for mcDESPOT also allows the calculation of standard relaxometry (T1\&2) maps from the same data.

\section{MRI Analysis}

The MR Images were first converted to NIFTI format from the manufacturer's proprietary format, and then were processed using a combination of FSL (Jenkinson et al., 2012), ANTs (Avants et al., 2011) and in-house C++ software utilizing the ITK library, available from https://github.com/spinicist/QUIT. The 


\begin{tabular}{|r|c|c|c|c|c|c|c|c|c|}
\hline Parameter & $\mathrm{T}_{\mathrm{M}}$ & $\mathrm{T} 2_{\mathrm{M}}$ & $\mathrm{T}_{\mathrm{IE}}$ & $\mathrm{T} 2_{\mathrm{IE}}$ & $\mathrm{T} 1_{\mathrm{FW}}$ & $\mathrm{T} 2_{\mathrm{FW}}$ & $\tau_{\mathrm{M}}$ & $\mathrm{MWF}(\%)$ & $\mathrm{FWF}(\%)$ \\
\hline Lower & 600 & 6 & 1000 & 40 & 1800 & 120 & 6 & 0.1 & 0 \\
Upper & 1000 & 20 & 1400 & 90 & 5000 & 2500 & 350 & 35 & 100 \\
\hline
\end{tabular}

Table 2. Lower and upper fitting bounds for the mcDESPOT parameters. The units are milliseconds for all parameters except the MWF and FWF.

processing pipeline consisted of several steps. The following operations were carried out in the native space of the acquired images.

1. A Tukey filter was applied in $k$-space to remove high frequency noise (Tagge et al., 2016). This is a common reconstruction step on clinical scanners but is not implemented on our system.

2. The B1 map was calculated from the AFI scan (Yarnykh, 2007). The single-component T1 map was then calculated from the SPGR data using the B1 map to correct the flip-angles. Because we acquired multiple flip-angles (required for mcDESPOT), we used a non-linear Levenberg-Marquadt algorithm instead of the common linearization method to fit the data. An initial value of $1 \mathrm{~s}$ was chosen for $T_{1}$ and no issues were observed with convergence to local minima.

3. T2 and off-resonance maps were calculated from the SSFP data, B1\&T1 maps using the DESPOT2FM method (Deoni, 2009). To improve the speed of the fitting procedure, instead of the original stochastic fitting method we used a bounded non-linear trust-region optimizer (Zhu et al., 1997). To ensure the global optimum was found four different starting points for off-resonance were ranging from $-1 /(2 * \mathrm{TR})$ to $+1 /(2 * \mathrm{TR})$ were tried. The starting point for T2 was set to T1/10. Yarnykh and Yuan (2004) lists values of T2/T1 ranging from 0.044 to 0.065 in various in-vivo and ex-vivo samples, but we observed fewer fitting failures using a larger initial value.

4. The mcDESPOT parameter maps, consisting of the Myelin Water Fraction (MWF), Intra-Extra cellular Water Fraction (IEWF), Free Water Fraction (FWF), T1\&T2 values for each fractional component, and the myelin water residence time $\left(\tau_{M}\right)$, were calculated using a Gaussian-prior stochastic Region Contraction method (GRC) (Deoni and Kolind, 2014). The mcDESPOT model is known to be difficult to fit, particularly close to banding artefacts in bSSFP data (Lankford and Does, 2012; Hurley and Alexander, 2014). In order to stabilize the fit we applied some additional simple heuristics; the off-resonance value of each component was fixed to the value calculated from DESPOT2-FM and we weighted the residuals of the bSSFP data by $3 \sin ^{2}(\phi+\psi) / 4$, where $\phi$ is the phase-increment value and $\psi$ is the accrued phase due to off-resonance in each TR. This weighting scheme ensures that data in the banding artefacts of one phase-increment is ignored in preference to data from the other phase-increments. The fittings ranges for the mcDESPOT parameters were chosen by observing the single-component T1\&2 values in regions of white and grey matter and are given in table 2 .

5. FSL topup and eddy were used to remove distortion and eddy current artefacts in the raw diffusion data (Andersson et al., 2003; Andersson and Sotiropoulos, 2016). We did not acquire data with a reversed phase-encode direction as the manufacturer's sequence does not have this option. Instead, we synthesized a standard spin-echo image from the T1\&T2 maps using the TE/TR of the diffusion sequence. The resulting image had no distortion and diffusion weighting, and was given to topup as a $b=0$ image, which could then undistort the acquired diffusion images. The DTI parameter maps were then calculated and consisted of Mean Diffusivity (MD), Axial Diffusivity (AD), Radial Diffusivity (RD) and Fractional Anisotropy (FA) using FSL dtifit.

The mcDESPOT processing produces ten separate parameter maps (ignoring the $B_{0}$ and $B_{1}$ parameter maps that correct for field inhomogeneities). However the MWF, IEWF and FWF are defined as fractions that must sum to one, and so are not independent parameters. Hence of these only the MWF was used for statistical analysis. Of the remaining parameters the myelin water residence time $\tau_{\mathrm{M}}$ could potentially be an indicator of myelin sheath integrity. However, as will be shown below the current mcDESPOT methodology cannot reliably fit this parameter, so we did not analyse it further. 


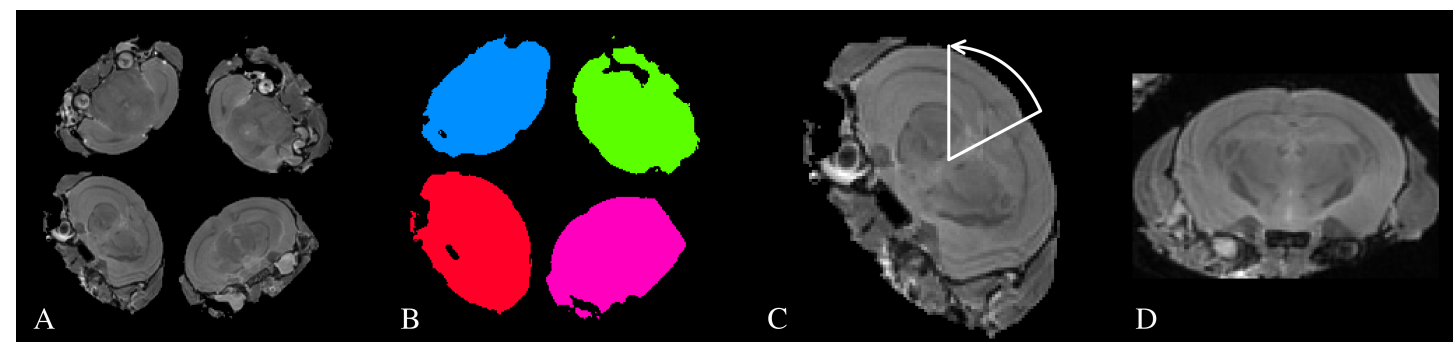

Figure 1. A schematic of the process used to automatically identify and re-orient each individual subject. A - A structural image showing how the subjects were arranged in the scanner B - The four largest connected components are labelled as the individual subjects. C - The Center-of-Gravity of each subject is identified, and a line drawn between the $\mathrm{CoG}$ and the origin. This defines the approximate rotation angle required for re-orientation. D - An individual subject after re-orientation and rigid registration to the atlas.

The following procedure was then used to split the images into individual subjects and register them to a common space:

1. The structural scan was bias-field corrected (Tustison et al., 2010), thresholded and the four largest connected-components were identified. These components were used as masks to separate each subject from the others. The center-of-gravity of each image was then calculated. Because the subjects were scanned in a consistent orientation (with the base of the brain towards the center of the sample tube), we calculated a simple rotation around the tube axis and translation that moved the subjects to the center of the field of view and oriented them in a standard manner. A simple rigid registration was then performed between each subject and an atlas image (Dorr et al., 2008) to ensure all samples were approximately aligned. This process is illustrated in figure 1 .

2. A template image was constructed using the 3D FSE and FA images from all subjects in the study (Avants et al., 2010). The resulting 3D FSE template was then non-linearly registered to the atlas image. Including the FA maps in the registration process improved the alignment of the external capsule between groups (see discussion).

3. All subjects were non-linearly registered to the study templates using their FSE and FA images. Logarithmic Jacobian determinants were calculated from the inverse warp fields in standard space to estimate apparent volume change. The transforms from native to study template, and from study to standard space were concatenated and applied to all relaxometry and DTI parameter maps to align them to the template. These images were resampled to match the voxel size of the template using a Gaussian interpolator. The full-width half-maximum of the interpolator was set to $100 \mu \mathrm{m}$ for the relaxometry data and $125 \mu \mathrm{m}$ for the DTI, due to their differing acquisition voxel sizes.

4. A brain parenchyma mask was created from the atlas labels by excluding CSF regions. The inverse transforms from the atlas to the study template and from the study template to each subject were applied to calculate the brain volume of each subject.

A group analysis was then carried out on all relaxometry maps, DTI maps and the Jacobian deteriminant images with permutation tests and Threshold-Free Cluster Enhancement (TFCE) using FSL randomize (Smith and Nichols, 2009; Winkler et al., 2014). The brain volume estimates were included as a regressor of no interest in the design matrix when analysing the Jacobian determinants, but not for the parameter maps. Animal weights and total brain volumes were compared using a separate two sample $\mathrm{t}$-Test assuming unequal variance.

\section{Histology}

After imaging the brains were removed from the skulls and cryoprotected by immersion in $30 \%$ sucrose for at least 72 hours. $20 \mu \mathrm{m}$ thick sections were cut in 12 series on a cryostat, collected onto chromegelatin coated slides and stored at $-20^{\circ} \mathrm{C}$. Immunohistochemistry was performed on two of the series with washes between each step. After rehydrating in Tris buffer (TBS) for $3 \times 5$ minutes endogenous peroxidase activity was blocked by applying $1 \%$ hydrogen peroxide $\left(\mathrm{H}_{2} \mathrm{O}_{2}\right)$ in TBS for 30 minutes at 
room temperature (RT), followed by a non-specific binding block with $10 \%$ skimmed milk powder in TBS with $2 \%$ Triton-X (TBS-X) for two hours at RT. Sections were then incubated in primary antibodies for either microglia (rabbit anti-Iba-1, 1:2000, 019-19741, Alpha Laboratories) or myelin basic protein (rat anti-MBP, 1:1000, ab7349, Abcam) diluted in 5\% skimmed milk powder in TBS-X overnight at $4^{\circ} \mathrm{C}$. Following three washes in TBS-X sections were incubated in either biotinylated goat anti-rabbit or anti-rat antibody diluted in 5\% skimmed milk powder in TBS-X (1:1000; BA-1000 and BA-9400 respectively, Vector Laboratories Ltd) for two hours at RT followed by incubation in avidin-horseradish peroxidase complex (Vectastain ABC Elite, PK-6000, Vector Laboratories) for one hour.

Immunoreactivity was visualized by incubating sections in $0.05 \%$ diaminobenzidine and $0.01 \% \mathrm{H}_{2} \mathrm{O}_{2}$ for up to five minutes with exact timing being determined by the depth of colour of the sections. Sections were then rinsed in TBS and dehydrated in increasing concentrations of industrial methylated spirits (IMS) followed by xylene before cover-slipping with DPX mounting medium (Sigma Aldrich, UK).

A third series was stained with Luxol Fast Blue to visualize myelin. Sections were placed in a 50/50 IMS/Histoclear solution overnight to remove fat from the tissue and then hydrated in 95\% IMS. Sections were then placed into Luxol Fast Blue solution at $56^{\circ} \mathrm{C}$ overnight, followed by rinses in $95 \%$ IMS and distilled water before differentiating in lithium carbonate for 30 seconds followed by 30 seconds in $70 \%$ IMS and a rinse in distilled water. Differentiation was then checked microscopically and differentiation steps repeated until grey matter was clear and white matter well defined. Once completed, sections were dehydrated in increasing concentrations of IMS followed by xylene before cover-slipping with DPX mounting medium. For analysis of all three stains we used three sections approximately $-1.58 \mathrm{~mm}$, $-1.82 \mathrm{~mm}$ and $-2.06 \mathrm{~mm}$ posterior from Bregma.

Iba1 stained sections were analysed with a Zeiss Axioskop2 MOT microscope and design-based optical fractionator probe in Stereoinvestigator software (v11.03.1, MBF Bioscience). An ROI was drawn over the corpus callosum, cortex above and hippocampus below covering an area of $20 \mathrm{~mm}^{2}$ in each section using a PlanApo 4x objective as shown in figure 7. Microglia population estimates were obtained using systematic random sampling with a sampling grid of $300 \times 300 \mu \mathrm{m}$ (Gundersen coefficient of error $<0.1)$ and a counting frame of $50 \times 50 \mu \mathrm{m}$. Section thickness was manually defined as $15 \mu \mathrm{m}$ with a dissector height of $14 \mu \mathrm{m}$ and guard zones of $0.5 \mu \mathrm{m}$ at the top and bottom of each frame. All microglia falling with the bounds of the counting frame and not touching the exclusion boundaries were counted using a PlanApo 40x objective at the monitor. Cell density was calculated as the ratio of the population estimate to the volume of the ROI, estimated using the Cavalieri principle (West et al., 1991).

Sections stained for MBP and Luxol Fast Blue were analysed using thresholding techniques. The MBP sections were first pseudocoloured in Aperio Imagescope (v12, Aperio Technologies Inc.) where staining in each pixel of the corpus callosum ROI was categorised into one of three levels and a snapshot at $\times 4$ magnification was then analysed by ImageJ $(\mathrm{v} 1.50 \mathrm{~b}, \mathrm{NIH})$ software. The analysis included removing of background and measuring the percent coverage of the appropriate peak corresponding to corpus callosum staining. The same method was applied to LFB but omitting the pseudocolouring step and using Otsu threshold parameters in ImageJ. All results were statistically analysed in Prism v6.07 (GraphPad Software Inc.) using unpaired T-tests.

\section{RESULTS}

The MR parameter maps and statistic images are available online (DOI: 10.6084/m9.figshare.3495848).

At the end of treatment the mean weight of the control and cuprizone groups were $27.5 \pm 2.5 \mathrm{~g}$ and $21.8 \pm 1.2 \mathrm{~g}$ respectively, which were significantly different when assessed with a two-tailed T-test $(p=0.0004)$. However the mean brain volumes were $387.8 \pm 10.1 \mathrm{~mm}^{3}$ and $381.5 \pm 9.0 \mathrm{~mm}^{3}$, which was not a significant difference $(p=0.26)$.

A single slice through the quantitative relaxometry parameter maps for a single control mouse is shown in figure 2 . White matter and grey matter are clearly distinguishable in the single-component maps. The T2 map better distinguishes the hippocampus and third ventricle at the base of the brain. The Myelin Water Fraction is clearly visible in the expected regions, with values of approximately $20 \%$ in the corpus callosum, slightly higher in the cerebral peduncles and optic tract and less than 5\% in grey matter regions. The Intra-Extra Cellular Water Fraction is approximately the inverse of the MWF, but a large amount of Free Water is indicated in the third ventricle.

The myelin T1\&2 maps are fairly flat across the brain, indicating that the fitting routine finds fairly consistent values for these parameters. The exception to this is that the $\mathrm{T} 2$ of myelin water in the cerebral 


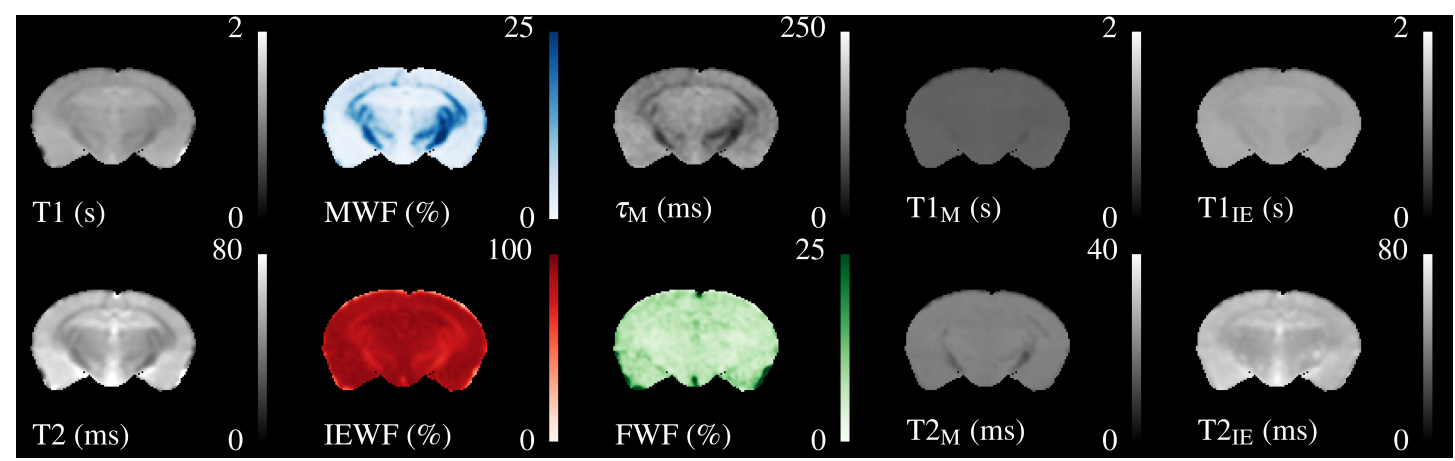

Figure 2. Illustrative relaxometry maps for a control mouse. Single-component T1\&2 maps are in the left-most column. There are ten mcDESPOT parameter maps - T1\&2 and the fractional amount of three water pools (Myelin, Intra-Extra cellular and Free), and the myelin residence time $\left(\tau_{M}\right)$. WM tracts are clearly visible in the MWF map. The T1\&2 maps of the Free Water (CSF) pool have been omitted.

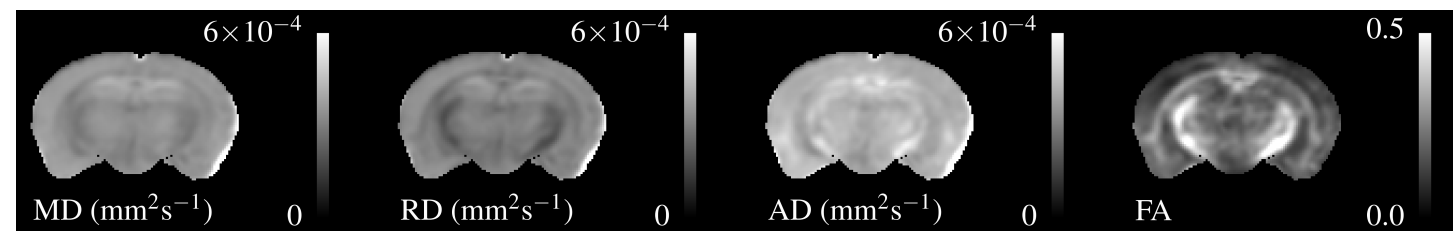

Figure 3. Single subject maps of the four DTI parameters - Mean, Radial and Axial Diffusivity and Fractional Anisotropy). These have lower resolution than the relaxometry data but the WM tracts are still visible. $\mathrm{MD}, \mathrm{AD} \& \mathrm{RD}$ all show similar contrast, but the absolute value of $\mathrm{AD}$ is higher than $\mathrm{RD}$.

peduncles and optic tract appear to be lower than that found in the corpus callosum. The T2 of the IE-water shows some differences between white and grey matter, but less than is found in the single-component T2 map. Although the myelin residence time ( $\tau_{M}$, defined as the mean time a water molecule stays in the myelin component before exchanging to the IE-pool) shows good contrast, it must be remembered that this parameter is not well defined outside of white matter tracts where there is close to $0 \%$ MWF (see below).

Similarly, figure 3 shows the DTI parameters from a single control mouse. These appear more blurred than the relaxometry maps due to the larger acquisition voxel size and interpolation FWHM, however there is still contrast between white and grey matter. AD is visibly higher than RD particularly in the hippocampus. FA shows good contrast between grey and white matter, especially in the cerebral peduncles and optic tract.

Figure 4 shows three axial slices through the group average T1, T2, MWF \& DTI maps at the level of the striatum, corpus callosum and arbor vitae of the cerebellum. The control group is presented on the left of each slice and the cuprizone group average on the right. Increases in T1\&2 are obvious in the corpus callosum and cerebellum, with a corresponding decrease in MWF. T2 increases are also visible outside of the white matter tracts. Similar effects are present for MD, RD, and AD, while changes in FA are less evident.

Figure 5 overlays the study template with the difference in group means for all parameters, thresholded at FWE-corrected $p<0.05$. At this threshold a strong decrease in MWF and corresponding increases in T1\& 2 of around $100 \& 20 \mathrm{~ms}$ respectively can be detected in the corpus callosum and arbor vitae. The detected regions of $\mathrm{T} 2$ change are much larger than for $\mathrm{T} 1$, and extend into the cortical and subcortical grey matter, including the sensorimotor cortex and the dorsal hippocampus. Increases in all diffusivities are also evident in corpus callosum, cerebellum, and thalamus. Changes in FA are restricted to a decrease directly in the splenium of the corpus callosum and an increase directly above, a decrease in the cingulate cortex and isolated decreases below the arbor vitae.

Regions of both volume increase and decrease were found in the TBM data. Large increases in volume were found in the splenium of the corpus callosum, external capsule, and inferior parts of the arbor vitae. These correspond well to areas of change indicated in the T1 and MWF maps. Decreases were found in 


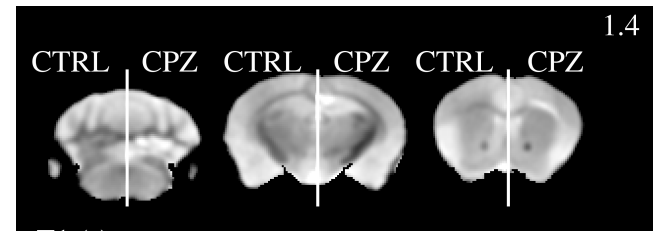

T1 (s)

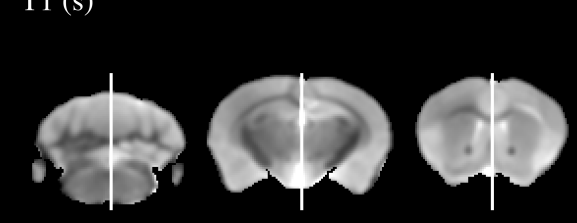

0.8

80

T2 (ms)
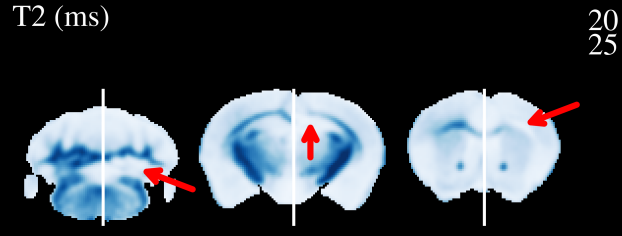

MWF (\%)

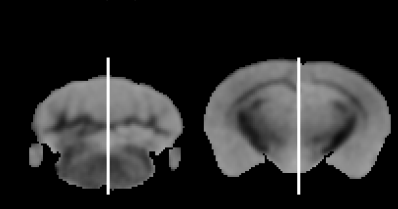

$\tau_{\mathrm{M}}(\mathrm{ms})$
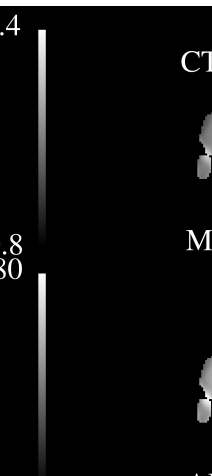

$\mathrm{MD}\left(\mathrm{mm}^{2} \mathrm{~s}^{-1}\right)$

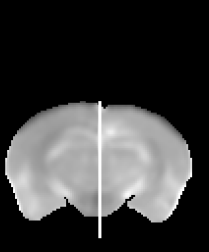

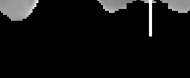

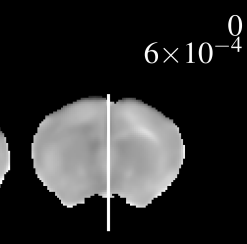

$\mathrm{AD}\left(\mathrm{mm}^{2} \mathrm{~s}^{-1}\right)$

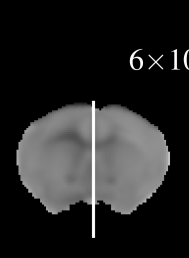

$6 \times 10^{-4}$

Figure 4. The mean control maps (left) compared against cuprizone (right) in three slices. Decreased MWF (red arrows) is obvious in WM. Similar changes are visible in other maps except for FA where the corpus callosum is visible in cuprizone animals (green arrows)

the cortex, striatum, dorsal hippocampus and fimbria, which do not appear to overlap with changes in the quantitative parameters. For reasons of space not all these regions are shown in 5, but can be viewed in the downloadable results.

Figure 6 shows histology slices for the LFB and MBP stains at approximately the same position as the MRI slices in figures $4 \& 5$, again with a control animal on the left and a cuprizone animal on the right. Decreases in both stains are clearly evident in the cuprizone animal, marked by arrows. In the corpus callosum and external capsule the MBP stain appears to show a decrease towards the edges of the tract, with MBP still present in the center. The cerebellar white matter is demyelinated in areas surrounding the cerebellar nuclei.

Figure 7 shows equivalent slices through the Iba1 staining for microglial activation. Zoomed areas are marked with boxes, showing the distinct shape of activated microglia in the cuprizone animal in the same areas that show demyelination in the LFB and MBP stains. Microglial activation appears less dense in the cerebellar nuclei compared to the corpus callosum. Figure 8 shows the quantitative histology results in the corpus callosum. Significant decreases in LFB and MBP staining, and increases in the number of Iba1 positive cells were found in the ROIs used. For LFB the mean intensities for control and cuprizone were $73.0 \pm 4.3$ and $26.8 \pm 3.4$ respectively $(p=0.0001$ ), for MBP they were $46.6 \pm 5.5$ and $26.8 \pm 3.4$ ( $p=0.0076)$ while for Iba1 the population counts were $4775 \pm 420$ and $11963 \pm 1513(p=0.0039)$.

Figure 9 shows a single slice of the Co-efficient of Variation $(\mathrm{CoV})$ for selected parameter maps. The CoV for $\mathrm{T} 1$ is excellent, and is less than 5\% throughout almost the entire parenchyma, while $\mathrm{T} 2$ is marginally worse. The CoV of MWF is highly region-dependent. In GM it is consistently above $10 \%$ and approaches $30 \%$ is some areas. This is perhaps expected given the low $(<5 \%)$ absolute value of MWF in these regions. However, even in WM tracts the CoV is generally close to $10 \%$ and does not fall below $5 \%$. The $\mathrm{CoV}$ of $\tau_{\mathrm{M}}$ shows that this parameter is difficult to fit. Counter-intuitively, in GM areas the CoV appears low while in WM areas it is high. However, it must be remembered that in GM areas there is close to $0 \% \mathrm{MWF}$, so here the fitting procedure simply converges to the center of the fitting range. In $\mathrm{WM}$ areas, where there should be sufficient MWF to fit a valid $\tau_{\mathrm{M}}$, the CoV map increases indicating that 


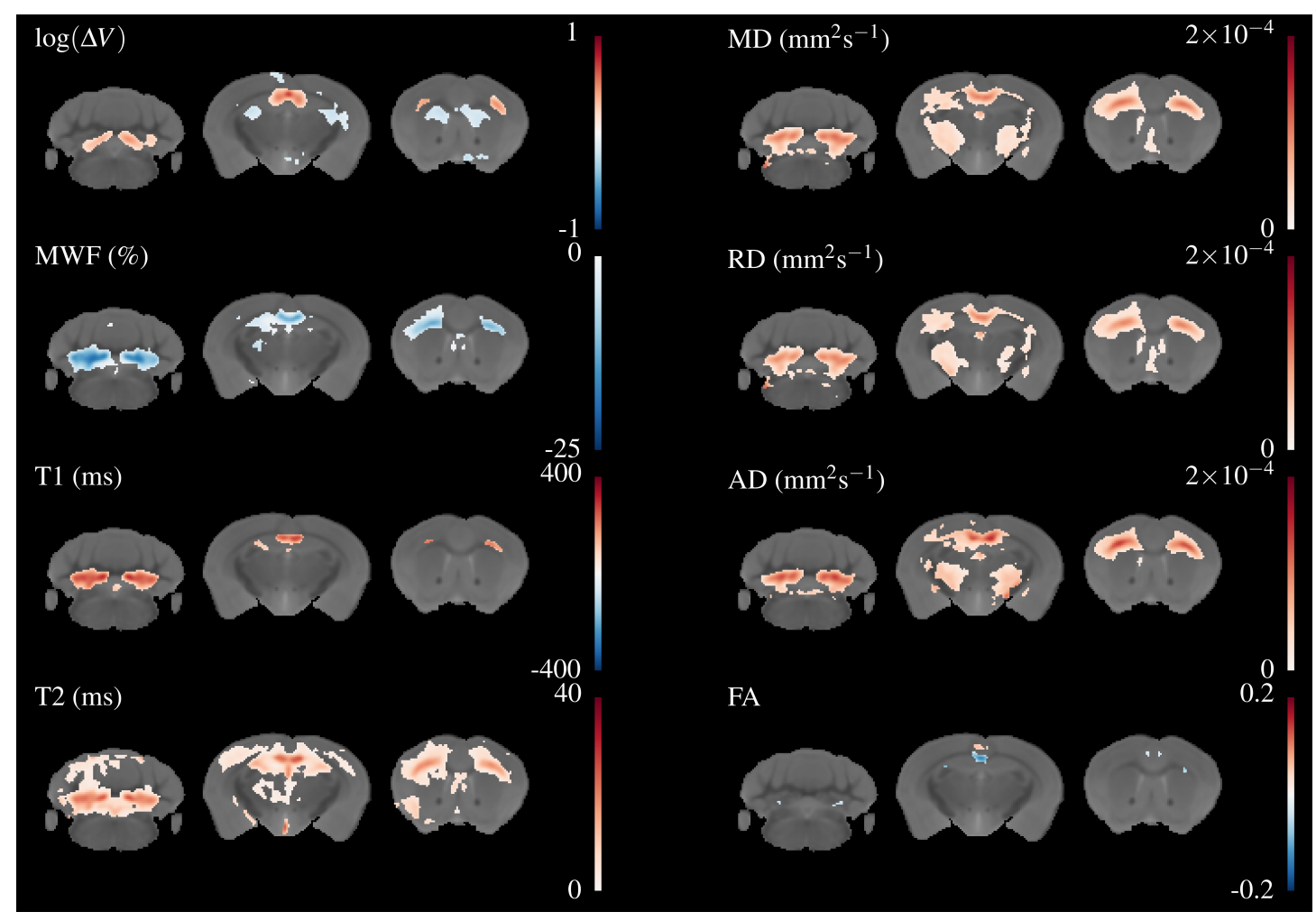

Figure 5. Differences in the group means of the volume change and quantitative parameters overlaid on the study template, thresholded at FWE-corrected $p<0.05$. The areas of significant change differ for each parameter, indicating different sensitivity and specificity to demyelination and inflammation.

there is insufficient information to fit this parameter correctly.

For the DTI parameters the diffusivity parameters have a mostly acceptable CoV $(<10 \%)$ that increases slightly in WM regions. We attribute this to partial volume effects and residual mis-registrations arising from the large voxel size in the anterior-posterior direction for the diffusion acquisition. FA has a high $\mathrm{CoV}$ that is above $10 \%$ in much of the parenchyma.

\section{DISCUSSION}

In this experiment we aimed to demonstrate the use of mcDESPOT in the cuprizone model, with images acquired over the entire brain, and compare the sensitivity and specificity of multiple quantitative MR methods to demyelination. However, the presence of inflammation in the cuprizone model is a significant potential confound that has not been adequately discussed in previous MR literature.

\section{Validation of mcDESPOT sensitivity to myelination}

A major aim of this study was to provide a pre-clinical validation of the MWF as measured by mcDESPOT as sensitive and specific to myelination state. In this regard the study can be regarded as success, principally because in contrast to Thiessen et al. (2013) we found a non-zero MWF in healthy control animals, and then observed a decrease in MWF in cuprizone treated mice.

However there are some important caveats to this apparent success. The first is that it is not immediately clear what advantages mcDESPOT brings over conventional relaxometry, given the extra acquisition time and extensive processing required to produce the parameter maps. As shown in figure 5 , the regions of significant change detected in $\mathrm{T} 1$ are most constrained to white matter, the regions of $\mathrm{T} 2$ change extend a long way into the GM, and MWF is somewhere in-between. No regions of significant change were detected in the MWF that were not also detected in either the T1 or T2 map. Due to the co-localisation of inflammation with demyelination in this model, it is difficult to disentangle the impact of these different mechanisms to the MR parameters. 

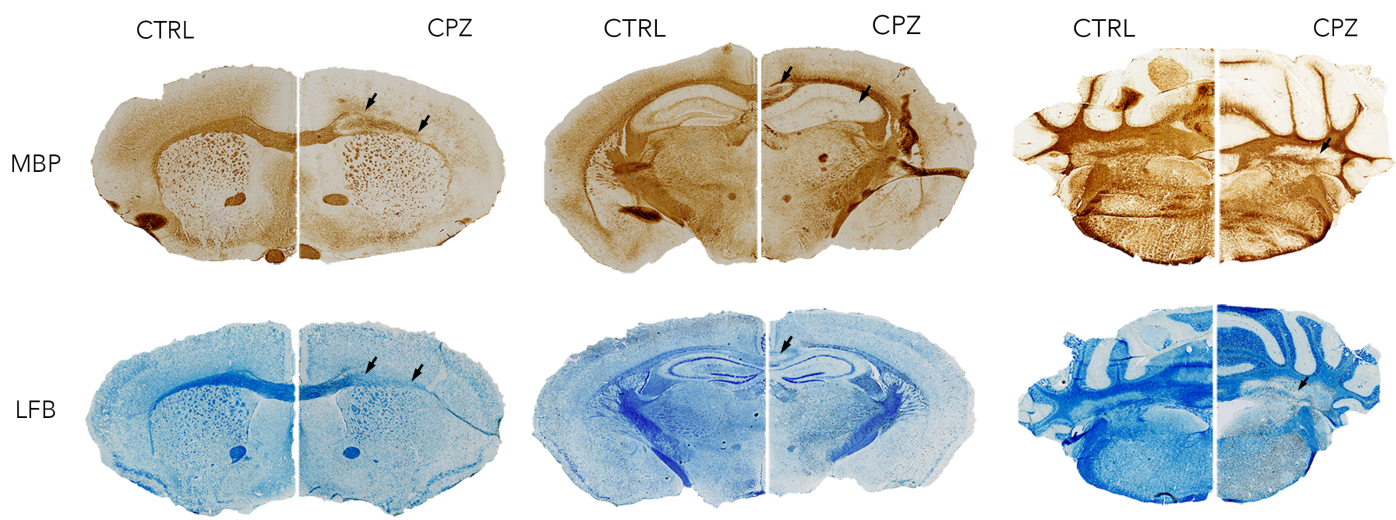

Figure 6. Representative histological sections stained with LFB and for MBP at approximately the same levels as the MRI. Again, a control animal (CTRL) is presented on the left of each slice and one treated with cuprizone (CPZ) on the right. Widespread decreases in both stains are marked with arrows in the corpus callosum. Distortion of white matter tracts is also evident.
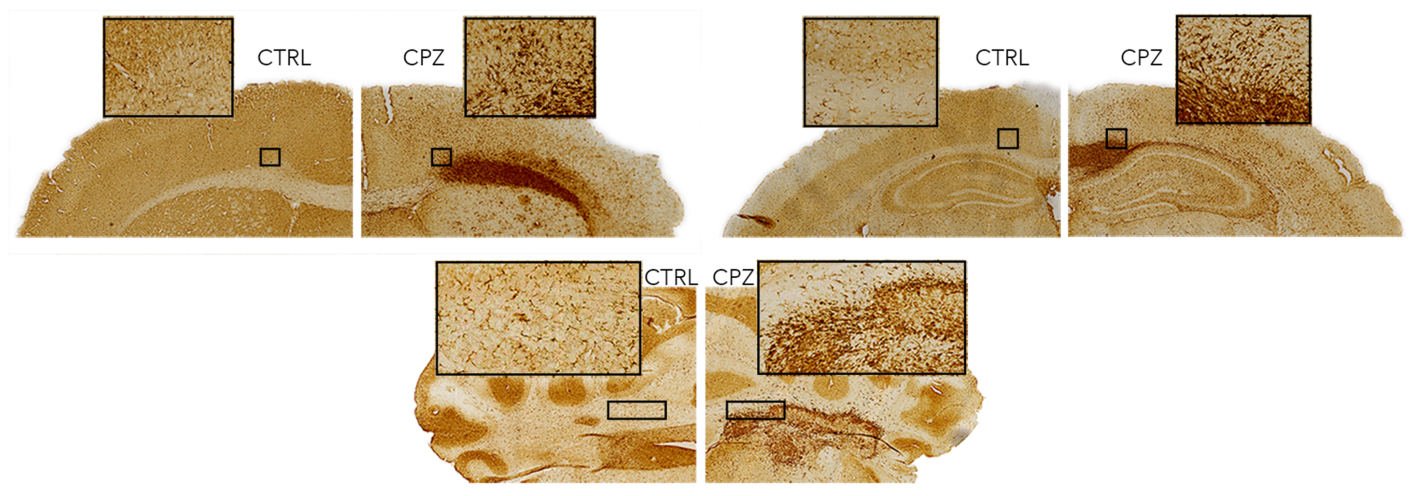

Figure 7. Representative histological sections stained for Iba1. Activated microglia (zoomed boxes) can be seen in the same regions where LFB and MBP staining indicate demyelination.
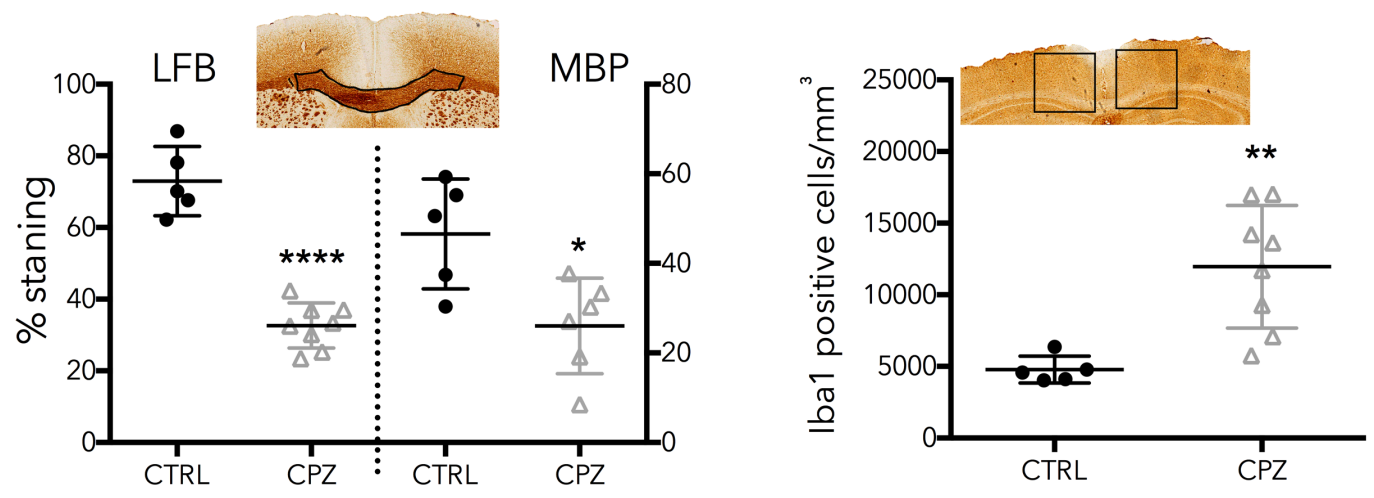

Figure 8. Quantitative histology results for the LFB, MBP and Iba1. ROIs are indicated on the histology images. Significant differences were found for all three stains, confirming the model functioned correctly. 


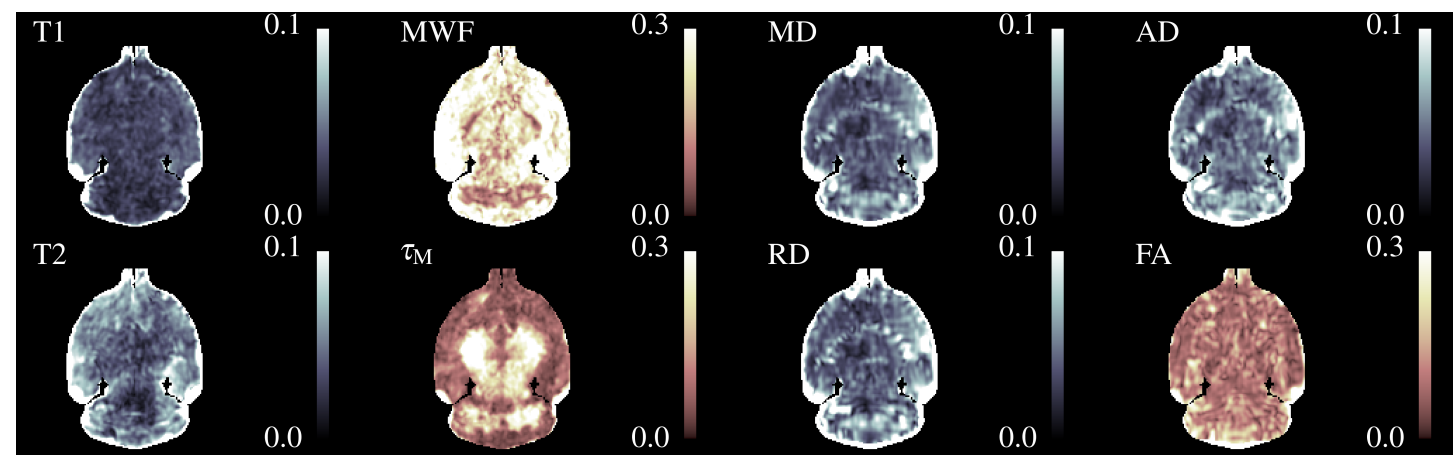

Figure 9. A coronal slice through the Co-efficient of Variation maps for selected parameters. The colourmap for MWF, $\tau_{\mathrm{M}}$ and FA was chosen to emphasise the different color scale. Briefly, the CoV is smallest for T1\&2, MD, RD \& AD and highest for FA \& MWF.

Moreover, because the MWF is defined as a fraction of total water in a voxel, it is obvious that a change in the absolute amount of IE-water will by definition change the MWF, although there has been no change in the absolute amount of myelin water. This means that by definition the MWF can only be sensitive and not specific to myelination state. As currently formulated, due to the need to normalize intensities between the SPGR and bSSFP acquisitions, mcDESPOT cannot be adapted to image absolute myelin and IE-water content, so further work is clearly needed in this area.

The final caveat is that the mcDESPOT model is difficult to fit correctly, and this has been remarked upon elsewhere (Lankford and Does, 2012; Zhang et al., 2014; Bouhrara and Spencer, 2015; Bouhrara et al., 2015). We found a lower MWF than has been reported for in-vivo mcDESPOT human studies, where typical values in WM are over 20\% rather than the 10-20\% reported here. These lower values are close to those reported by MET2 studies in human in-vivo and ex-vivo studies. However we found these values to be sensitive to the fitting ranges used, in particular for $\tau_{\mathrm{M}}$, the residence time of water in myelin. As described above, this parameter is meaningless in GM regions and simply converges to the center of any chosen fitting range.

In white matter, where the residence time is well defined, we found values below $50 \mathrm{~ms}$. This is significantly shorter than that reported for human studies. This could be attributed to species differences, or the process of paraformaldehyde fixation, which is known to disrupt biological membranes and introduce holes into otherwise impermeable structures (Zhang et al., 2012). This will increase the rate at which water can move between the myelin and IE-water pools. Hence we do not think the low residence times are artefactual, but they do conflict with both the widely used MET2 model and the results of Bouhrara et al. (2015), which do not include any exchange effects because it has been assumed that exchange is slow in relation to T2. Given the high $\mathrm{CoV}$ for $\tau_{\mathrm{M}}$ these values should be treated with some caution and further work is needed to improve the accuracy with which this parameter can be extracted from mcDESPOT data.

\section{The cuprizone model}

The above results show that the effect of cuprizone treatment is not limited to the splenium of the corpus callosum. To the best of our knowledge this is the first study to use MRI to show extensive cuprizoneinduced changes throughout both the cerebrum and cerebellum. In our data the effect of demyelination was particularly striking in the arbor vitae, which contains the deep cerebellar nuclei surrounded by heavily myelinated tracts. Cerebellar demyelination and inflammation has been previously shown in histological examinations, although changes in the cerebellar cortex were less pronounced and delayed (Groebe et al., 2009; Skripuletz et al., 2010). A recent experiment where rats were administered cuprizone and then serially imaged suggested that demyelination started in the cerebellum and progressed forwards in the brain (Oakden et al., 2016).

We found no difference in total brain volume, despite confirming that cuprizone causes significant weight loss (Nystad et al., 2014). We did not test for behavioural changes in our model. It is well known that despite causing profound changes in the brain, cuprizone treatment causes no large behavioural changes and only subtle motor and cognitive deficits, which would require specialized equipment to test 
for (Skripuletz et al., 2011).

The effect of cuprizone in the principal areas of the arbor vitae, splenium and external capsule is very strong in our data. In the arbor vitae the change in T1 is approximately $20 \%$, and in T2 it approaches $50 \%$. This means that despite our low group sizes the statistical analysis appears extremely robust, with many voxels meeting the $p<0.05$ threshold even using the conservative FWE multiple comparisons correction. $\mathrm{T} 2$ but not $\mathrm{T} 1$ detects changes in grey matter regions such as the cortex and thalamus, which are known to also be affected by cuprizone (Gudi et al., 2009; Fjær et al., 2013; Goldberg et al., 2015). Even more widespread changes in T2 could be seen using FDR instead of FWE correction (available in the on-line dataset), whereas changes in $\mathrm{T} 1$ remain restricted to areas that are heavily myelinated in healthy animals.

We also detected changes in several quantitative parameters in the hippocampus, where myelination and inflammatory changes have been well characterised by histology (Skripuletz et al., 2011; Goldberg et al., 2015). This is again a region which most imaging studies have neglected to either analyze or report. Our results indicate the possibility that previous imaging studies to document the longitudinal profile of the cuprizone model have been missing important areas of the brain, and inclusion of these areas in future studies is necessary.

It was beyond the scope of this paper to perform a fully quantitative histological evaluation of all brain areas in which the MRI changes were detected. Many studies to date have shown extensive histological and immunohistochemical changes due to cuprizone treatment, which match both our qualitative (Figs 6 \& 7) and quantitative results (Fig 8). We limited our myelination quantification with LFB and MBP to the key areas of rostral corpus callosum, and microglial assessment with Iba1 to the surrounding cortex and hippocampus. As expected, these showed a robust and significant decrease in myelination in cuprizone mice, and a profound increase in number of activated microglia. It is also clear from qualitative observations of histological slides that areas of demyelination are spread throughout the brain and particularly salient at the external capsule, hippocampus and arbor vitae. The affected white matter areas are accompanied and surrounded by clearly increased Iba1 staining and visibly enlarged microglial cell bodies, indicating ongoing microglial hypertrophy and hyperplasia. The colocation of demyelination and inflammation in the cuprizone model means that it is difficult to rule out a contribution from inflammation to changes in the MR parameters.

We believe this study is the first to estimate brain volumes and apparent volume change in the cuprizone model. Localized volume changes (both increases and decreases) were widespread and extended to regions that showed no change in relaxometry or DTI parameters. Volume increases were located in the corpus callosum, dorsal hippocampus, arbor vitae and sensorimotor cortex. These were corroborated by histology as apparent enlargement and distortion of WM tracts, reflecting previously reported underlying axonal swelling, damage and cellular infiltration (Song et al., 2005; Xie et al., 2010). In addition we also found bilateral decreases in the frontal, cingulate and retrosplenial cortices as well as the caudate putamen and several other subcortical grey matter areas.

The lack of change in FA (discussed further below) was beneficial for this study as this parameter could be used to improve the registration process. Initially, we followed standard practice and used only the 3D FSE image for template creation and registration. However, due to the strong effect of cuprizone the FSE contrast in several white matter regions is reversed compared to control animals. This led to subtle misalignments between the two groups, principally of the external capsule, resulting in nonsensical changes in volume and FA outside WM tracts. Incorporating the FA maps into the registration correctly aligned the external capsule in the cuprizone group.

This demonstrates a difficulty in using current automated registration methods, as fundamentally they assume that all input images are equivalent and this is clearly not the case when gross pathology or even subtle changes in T1\&2 are present (Cousins et al., 2013). Although non-linear deformation algorithms include regularisation methods to prevent excessive volume changes (van Eede et al., 2013), this was not sufficient in our data to prevent mis-registrations. However, incorporating a parameter that did not demonstrate gross changes between groups (FA) stabilised the registration and yielded a high quality result.

\section{Diffusion measurements in the cuprizone model}

DTI has become an extremely widespread method of assessing WM health in clinical studies (Jones et al., 2013), but interpretation of the diffusivity and FA parameters can be difficult. In particular, RD and FA are often assumed to be a marker of myelin "integrity" (Song et al., 2005; Wheeler-Kingshott and Cercignani, 


\begin{tabular}{|c|c|c|c|c|c|}
\hline Citation & $\mathrm{N}$ & $\begin{array}{l}\delta / \Delta \\
(\mathrm{ms})\end{array}$ & $\begin{array}{c}b \\
\left(\mathrm{~ms} / \mu \mathrm{m}^{2}\right)\end{array}$ & $\begin{array}{c}\text { In-/Ex- } \\
\text { Vivo }\end{array}$ & Results \\
\hline Song et al. (2005) & 6 & $4 / 11$ & 1.6 & In & $6 \mathrm{w}: \mathrm{RD} \uparrow$ \\
\hline Sun et al. (2006) & 6 & $10 / 25$ & 0.768 & In & $4 \mathrm{w}: \mathrm{AD} \downarrow, 6 \mathrm{w}: \mathrm{AD} \downarrow \mathrm{RD} \uparrow$ \\
\hline Wu et al. (2008) & $2 *$ & $*$ & 0.7 & In & $4 \mathrm{w}: \mathrm{AD} \downarrow R \mathrm{R} \uparrow$ \\
\hline Xie et al. (2010) & 6 & $8 / 25$ & 0.768 & In & $4 \mathrm{w}: \mathrm{AD} \downarrow$ \\
\hline Zhang et al. (2012) & 6 & $3 / 15$ & 1.0 & In & $4 \mathrm{w}: \mathrm{AD} \downarrow 6 \mathrm{w}: \mathrm{RD} \uparrow$ \\
\hline & & & 1.7 & Ex & $4 \mathrm{w}: \mathrm{FA} \downarrow \mathrm{RD} \uparrow, 6 \mathrm{w}: \mathrm{FA} \downarrow \mathrm{RD} \uparrow$ \\
\hline Chandran et al. (2012) & 30 & $3 / 10$ & 0.75 & In & $5 \mathrm{w}: \mathrm{FA} \downarrow$ \\
\hline Thiessen et al. (2013) & 7 & $6 / 14$ & 1.0 & Ex & $6 \mathrm{w}: \mathrm{FA} \downarrow \mathrm{AD}, \mathrm{RD} \uparrow$ \\
\hline Guglielmetti et al. (2016) & 30 & $5 / 12$ & $0.4-2.8$ & In & $3 \mathrm{w}: \mathrm{MD}, \mathrm{AD}, \mathrm{RD} \downarrow, 6 \mathrm{w}: \mathrm{MD}, \mathrm{AD}, \mathrm{RD} \uparrow$ \\
\hline Jelescu et al. (2016) & 30 & $2 / 16$ & $1.0 / 2.0$ & In & $6 \mathrm{w}: \mathrm{RD} \uparrow$ \\
\hline
\end{tabular}

Table 3. A summary of DTI protocols and findings in the acute phase of cuprizone treatment. $\mathrm{N}$ Number of diffusion encoding directions. Wu et al. (2008) did not report their $\delta / \Delta$ values, and used two directions oriented parallel and perpendicular to the corpus callosum instead of calculating the tensor. Results past 6 weeks omitted for brevity. The findings vary widely, but this is at least in part due to the different imaging protocols and treatment lengths.

2009; Janve et al., 2013). Our results indicate two issues; firstly that both RD and AD (and hence MD) are sensitive to demyelination, and that FA appears to be far less sensitive to changes in myelination than diffusivities.

There are conflicting literature results for diffusion changes in the acute phase of the cuprizone model, which are summarised in table 3 . Results from the chronic (greater than six weeks) phase of cuprizone treatment have been omitted, as these do not relate to our time point. However, the reported results also vary widely in diffusion protocols, importantly the number of diffusion directions and strengths. It is possible that earlier papers using only six directions could not correctly calculate the tensor correctly for tracts in all orientations. Some caution should be applied to interpretations comparing diffusion imaging from in-vivo and ex-vivo subjects, because as discussed by Zhang et al. (2012), diffusivity parameters may alter as a result of tissue fixation. Another contributory factor is different lengths of cuprizone treatment, as the levels of demyelination and inflammation in cuprizone undergo progressive change between four and six weeks of treatment (Skripuletz et al., 2011). In particular, at week four demyelination is incomplete whereas microglial activation is highest, but by week five demyelination is complete but microglial activation is decreasing. Hence, as discussed for relaxometry above, there are competing effects of inflammation and demyelination on diffusivity measurements which are hard to disentangle using this model. Recent work (Guglielmetti et al., 2016) supports this hypothesis, as they found an initial decrease of diffusivity after three weeks of cuprizone treatment, followed by an increase at six weeks.

The limited areas of change in FA detected in the cuprizone model were surprising. There is only one area of convincing change, a decrease directly at the splenium of the corpus callosum, with an increase directly above. Comparison to histology suggests that this region of the corpus callosum appears to distort, reducing the ordered nature of the axons. Smaller potential changes are located in the cingulate cortex and on the edges of the arbor vitae, but there are no widespread effects throughout WM tracts as for the other parameter maps. This suggests that FA is insensitive to the myelination state of WM, although this may be due to the increased variance in the FA measurements.

It was beyond the scope of this work to include an assessment of axonal damage via neurofilament histological staining. However recent work indicates that during the acute phase (less than six weeks) of cuprizone treatment axonal damage should be minimal (Schregel et al., 2012; Alme et al., 2015), and only starts to accumulate during the chronic phase (Lindner et al., 2009). This suggests that the changes we see in FA are due to changes in the extracellular matrix or organisation of axons, rather than outright damage.

\section{Comparison of DTI and relaxometry data}

Our results are broadly in line with those of Santis et al. (2014), who compared mcDESPOT and DTI in healthy human subjects. They found a low correlation between FA and measures of myelination. They 


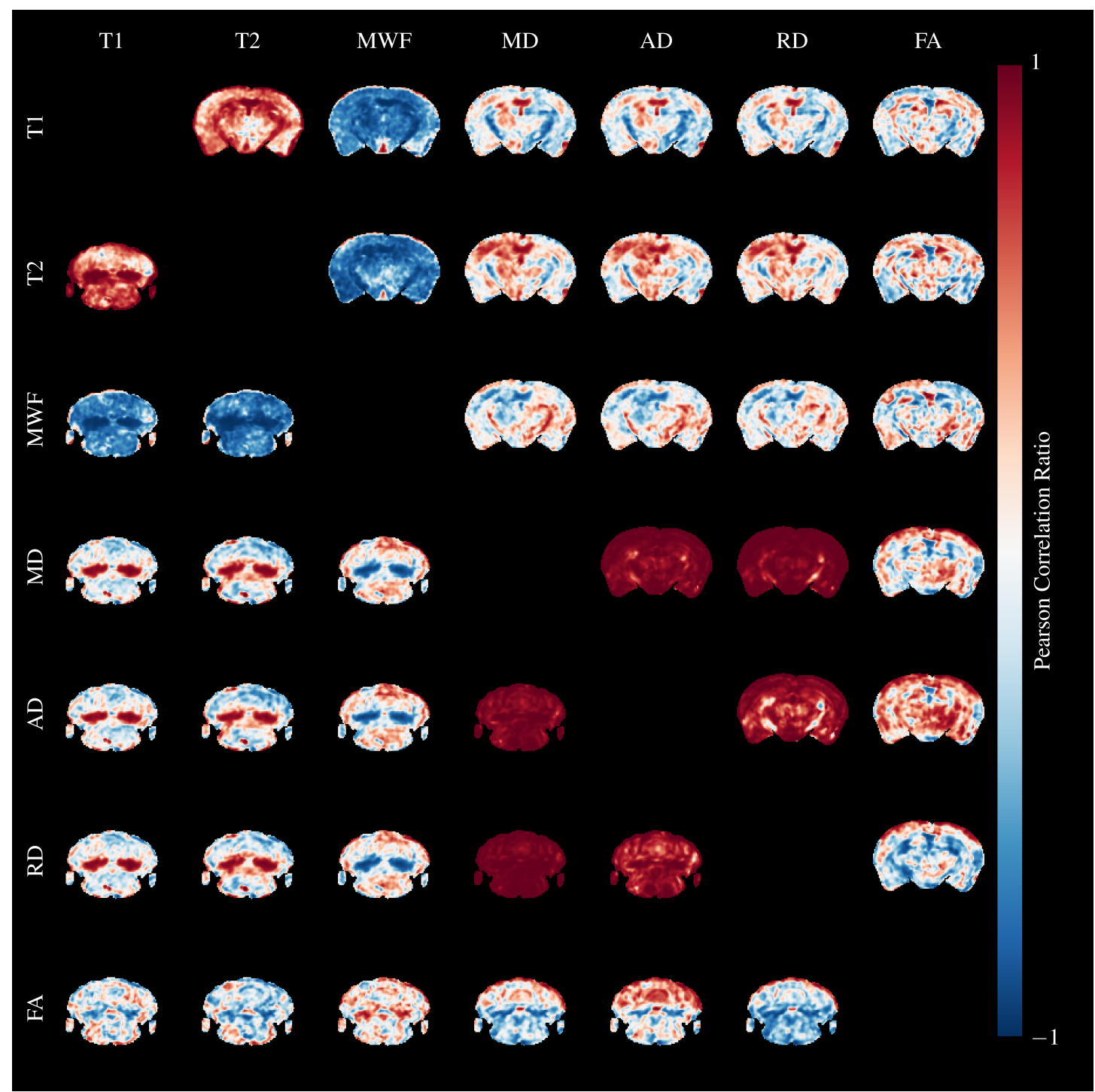

Figure 10. Pearson's Correlation Ratio between each pair of MR parameters. A slice through the external capsule is shown above the diagonal and a slice through the cerebellum below the diagonal. Correlations between parameters are very strong (but both positive and negative) in the arbor vitae and splenium. 
also found a similar trend of parameter variance, with T1 having the best performance and FA \& MWF the worst. They found that $\mathrm{T} 2$ measurements performed significantly worse than diffusivities, while we found it performed slightly better. We attribute this to the increased number of phase increments in our bSSFP acquisition, which we found markedly decreased banding artefacts.

We calculated Pearson's correlation ratio between the quantitative parameters across the entire brain and all mice using AFNI (Cox, 2012). Slices through the splenium and cerebellum are shown above and below the diagonal respectively in figure 10. Red colors indicate strong positive correlations, and blue indicates strong negative correlations. Perhaps unsurprisingly, the relaxometry parameters and diffusivities form two separate groups of highly correlated parameters.

T1\&2 are highly correlated in white matter regions, and particularly so where cuprizone acts, but are less strongly correlated in grey matter. They are both negatively correlated to MWF in similar patterns. Correlations between diffusivities and T1\&2 are poor except in areas where cuprizone has a strong effect. Correlations between FA and the other parameters are poor. MD, AD and RD are highly correlated in both grey and white matter, except for $\mathrm{AD}$ and RD in the cerebral peduncles, fimbria and optic tract. These white matter tracts are known to be resistant to cuprizone damage at five weeks (Goldberg et al., 2015), and we observed no qualitative changes in our histology in these regions. Hence the low correlations are likely a result of different white matter organisation in these regions compared to the highly ordered corpus callosum.

This analysis underscores the severity of the cuprizone model at five weeks - in the primarily affected regions, the difference between model and control is so complete that correlations across the entire dataset approach one, and we expect the same would hold true for a histological analysis. It would be useful to better understand the contribution of myelin and inflammation to the different MR parameters, similarly to how Desmond et al. (2016) developed a model of the contribution of myelin and metals to T1\&2. In our opinion, due to the strength of the correlations the single time point presented here is insufficient to build such a model. However, microglial activation is know to precede demyelination in the cuprizone model by approximately a week (Skripuletz et al., 2011), and is required for demyelination to occur (Praet et al., 2015). Hence a longitudinal study with several earlier time points should observe a differential build up of inflammation and demyelination, allowing the two effects to be separated.

\section{CONCLUSION}

This experiment demonstrates that T1\&2, the Myelin Water Fraction, and diffusivities are sensitive to demyelination but not necessarily specific, due to confounding co-localized inflammation in the cuprizone model. Fractional Anisotropy appears insensitive to myelination state. In addition we found that cuprizone causes localized volume changes in the mouse brain. Collectively these results show that whole brain acquisition and analysis is crucial to full understanding of the cuprizone model. We propose that similar methods would be beneficial when using MRI to study other preclinical models of neurodegeneration to better understand and refine the knowledge of brain pathology.

\section{ACKNOWLEDGEMENTS}

We acknowledge many useful discussions with Jonathan O'Muircheartaigh. We thank Professor Federico Turkheimer for reading the paper and making helpful suggestions.

\section{REFERENCES}

Alme, M. N., Nystad, A. E., Bø, L., Myhr, K.-M., Vedeler, C. A., Wergeland, S., and Torkildsen, Ø. (2015). Fingolimod does not enhance cerebellar remyelination in the cuprizone model. Journal of Neuroimmunology, 285:180 - 186.

Andersson, J. L., Skare, S., and Ashburner, J. (2003). How to correct susceptibility distortions in spin-echo echo-planar images: application to diffusion tensor imaging. NeuroImage, 20(2):870 - 888.

Andersson, J. L. and Sotiropoulos, S. N. (2016). An integrated approach to correction for off-resonance effects and subject movement in diffusion MR imaging. NeuroImage, 125:1063-1078.

Avants, B. B., Tustison, N. J., Song, G., Cook, P. A., Klein, A., and Gee, J. C. (2011). A reproducible evaluation of ANTs similarity metric performance in brain image registration. NeuroImage, 54(3):2033 -2044 . 
Avants, B. B., Yushkevich, P., Pluta, J., Minkoff, D., Korczykowski, M., Detre, J., and Gee, J. C. (2010). The optimal template effect in hippocampus studies of diseased populations. NeuroImage, 49(3):2457 2466.

Bouhrara, M., Reiter, D. A., Celik, H., Fishbein, K. W., Kijowski, R., and Spencer, R. G. (2015). Analysis of mcdespot- and cpmg-derived parameter estimates for two-component nonexchanging systems. Magnetic Resonance in Medicine, pages n/a-n/a.

Bouhrara, M. and Spencer, R. G. (2015). Incorporation of nonzero echo times in the SPGR and bSSFP signal models used in mcDESPOT. Magnetic Resonance in Medicine.

Cahill, L. S., Laliberté, C. L., Ellegood, J., Spring, S., Gleave, J. A., van Eede, M. C., Lerch, J. P., and Henkelman, R. M. (2012). Preparation of fixed mouse brains for MRI. NeuroImage, 60(2):933 - 939.

Chandran, P., Upadhyay, J., Markosyan, S., Lisowski, A., Buck, W., Chin, C.-L., Fox, G., Luo, F., and Day, M. (2012). Magnetic resonance imaging and histological evidence for the blockade of cuprizone-induced demyelination in C57BL/6 mice. Neuroscience, 202(0):446 - 453.

Cousins, D. A., Aribisala, B., Ferrier, I. N., and Blamire, A. M. (2013). Lithium, gray matter, and magnetic resonance imaging signal. Biological Psychiatry, 73(7):652 - 657. New Insights into the Treatment of Mood Disorders.

Cox, R. W. (2012). Afni: What a long strange trip it's been. NeuroImage, 62(2):743 - 747. 20 \{YEARS $\{\mathrm{OF}\}$ fMRI20 \{YEARS $\}\{\mathrm{OF}\}$ fMRI.

Deoni, S. C. and Kolind, S. H. (2014). Investigating the stability of mcDESPOT myelin water fraction values derived using a stochastic region contraction approach. Magnetic Resonance in Medicine.

Deoni, S. C. L. (2009). Transverse relaxation time (T2) mapping in the brain with off-resonance correction using phase-cycled steady-state free precession imaging. Journal of Magnetic Resonance Imaging, 30(2):411-417.

Deoni, S. C. L., Matthews, L., and Kolind, S. H. (2013). One component? Two components? Three? The effect of including a nonexchanging "free" water component in multicomponent driven equilibrium single pulse observation of T1 and T2. Magnetic Resonance in Medicine, 70(1):147-154.

Desmond, K. L., Al-Ebraheem, A., Janik, R., Oakden, W., Kwiecien, J. M., Dabrowski, W., Rola, R., Geraki, K., Farquharson, M. J., Stanisz, G. J., and Bock, N. A. (2016). Differences in iron and manganese concentration may confound the measurement of myelin from $\mathrm{r} 1$ and $\mathrm{r} 2$ relaxation rates in studies of dysmyelination. NMR in Biomedicine, pages n/a-n/a.

Dorr, A., Lerch, J., Spring, S., Kabani, N., and Henkelman, R. (2008). High resolution three-dimensional brain atlas using an average magnetic resonance image of 40 adult $\mathrm{C} 57 \mathrm{Bl} / 6 \mathrm{~J}$ mice. Neurolmage, 42(1):60 - 69 .

Dubessy, A.-L., Zujovic, V., Papeix, C., and Stankoff, B. (2014). Biotherapies in multiple sclerosis: A step toward remyelination and neuroprotection? Revue Neurologique, 170(12):770 - 778.

Falangola, M. F., Guilfoyle, D. N., Tabesh, A., Hui, E. S., Nie, X., Jensen, J. H., Gerum, S. V., Hu, C., LaFrancois, J., Collins, H. R., and Helpern, J. A. (2014). Histological correlation of diffusional kurtosis and white matter modeling metrics in cuprizone-induced corpus callosum demyelination. NMR in Biomedicine.

Fjær, S., B $\varnothing$, L., Lundervold, A., Myhr, K.-M., Pavlin, T., Torkildsen, Ø., and Wergeland, S. (2013). Deep gray matter demyelination detected by magnetization transfer ratio in the cuprizone model. PLOS ONE, 8(12):e84162.

Goldberg, J., Clarner, T., Beyer, C., and Kipp, M. (2015). Anatomical distribution of cuprizone-induced lesions in C57BL6 mice. Journal of Molecular Neuroscience, 57(2):166-175.

Groebe, A., Clarner, T., Baumgartner, W., Dang, J., Beyer, C., and Kipp, M. (2009). Cuprizone treatment induces distinct demyelination, astrocytosis, and microglia cell invasion or proliferation in the mouse cerebellum. The Cerebellum, 8(3):163-174.

Gudi, V., Moharregh-Khiabani, D., Skripuletz, T., Koutsoudaki, P. N., Kotsiari, A., Skuljec, J., Trebst, C., and Stangel, M. (2009). Regional differences between grey and white matter in cuprizone induced demyelination. Brain Research, 1283:127 - 138.

Guglielmetti, C., Veraart, J., Roelant, E., Mai, Z., Daans, J., Audekerke, J. V., Naeyaert, M., Vanhoutte, G., y Palacios, R. D., Praet, J., Fieremans, E., Ponsaerts, P., Sijbers, J., der Linden, A. V., and Verhoye, M. (2016). Diffusion kurtosis imaging probes cortical alterations and white matter pathology following cuprizone induced demyelination and spontaneous remyelination. NeuroImage, 125:363 - 377.

Hurley, S. A. and Alexander, A. L. (2014). Assessment of mcDESPOT precision using constrained 
estimation. In ISMRM, volume 22, page 3144.

Janve, V. A., Zu, Z., Yao, S.-Y., Li, K., Zhang, F. L., Wilson, K. J., Ou, X., Does, M. D., Subramaniam, S., and Gochberg, D. F. (2013). The radial diffusivity and magnetization transfer pool size ratio are sensitive markers for demyelination in a rat model of type III multiple sclerosis (MS) lesions. NeuroImage, 74:298 - 305 .

Jelescu, I. O., Zurek, M., Winters, K. V., Veraart, J., Rajaratnam, A., Kim, N. S., Babb, J. S., Shepherd, T. M., Novikov, D. S., Kim, S. G., and Fieremans, E. (2016). In vivo quantification of demyelination and recovery using compartment-specific diffusion MRI metrics validated by electron microscopy. NeuroImage, 132:104 - 114.

Jenkinson, M., Beckmann, C. F., Behrens, T. E., Woolrich, M. W., and Smith, S. M. (2012). FSL. NeuroImage, 62(2):782 - 790 .

Jones, D. K., Knösche, T. R., and Turner, R. (2013). White matter integrity, fiber count, and other fallacies: The do's and don'ts of diffusion MRI. NeuroImage, 73(0):239 - 254.

Lankford, C. L. and Does, M. D. (2012). On the inherent precision of mcDESPOT. Magnetic Resonance in Medicine.

Lee, J., Shmueli, K., Kang, B.-T., Yao, B., Fukunaga, M., van Gelderen, P., Palumbo, S., Bosetti, F., Silva, A. C., and Duyn, J. H. (2012). The contribution of myelin to magnetic susceptibility-weighted contrasts in high-field MRI of the brain. NeuroImage, 59(4):3967 - 3975.

Lindner, M., Fokuhl, J., Linsmeier, F., Trebst, C., and Stangel, M. (2009). Chronic toxic demyelination in the central nervous system leads to axonal damage despite remyelination. Neuroscience Letters, 453(2): $120-125$.

Mackay, A., Whittall, K., Adler, J., Li, D., Paty, D., and Graeb, D. (1994). In vivo visualization of myelin water in brain by magnetic resonance. Magnetic Resonance in Medicine, 31(6):673-677.

Merkler, D., Boretius, S., Stadelmann, C., Ernsting, T., Michaelis, T., Frahm, J., and Brück, W. (2005). Multicontrast MRI of remyelination in the central nervous system. NMR in Biomedicine, 18(6):395-403.

Nave, K.-A. and Werner, H. B. (2014). Myelination of the nervous system: mechanisms and functions. Annual review of cell and developmental biology, 30:503-533.

Nystad, A. E., Wergeland, S., Aksnes, L., Myhr, K.-M., Bø, L., and Torkildsen, Ø. (2014). Effect of highdose 1.25 dihydroxyvitamin $\mathrm{d} 3$ on remyelination in the cuprizone model. APMIS, 122(12):1178-1186.

Oakden, W., Bock, N., Al-Ebraheem, A., Farquharson, M., and Stanisz, G. (2016). MRI of cuprizone induced demyelination in rat brain. In ISMRM, volume 24, page 1312.

Praet, J., Orije, J., Kara, F., Guglielmetti, C., Santermans, E., Daans, J., Hens, N., Verhoye, M., Berneman, Z., Ponsaerts, P., and Van der Linden, A. (2015). Cuprizone-induced demyelination and demyelinationassociated inflammation result in different proton magnetic resonance metabolite spectra. NMR in Biomedicine, 28(4):505-513. NBM-14-0226.R2.

Santis, S. D., Drakesmith, M., Bells, S., Assaf, Y., and Jones, D. K. (2014). Why diffusion tensor MRI does well only some of the time: Variance and covariance of white matter tissue microstructure attributes in the living human brain. NeuroImage, 89:35 - 44.

Schregel, K., Wuerfel née Tysiak, E., Garteiser, P., Gemeinhardt, I., Prozorovski, T., Aktas, O., Merz, H., Petersen, D., Wuerfel, J., and Sinkus, R. (2012). Demyelination reduces brain parenchymal stiffness quantified in vivo by magnetic resonance elastography. Proceedings of the National Academy of Sciences, 109(17):6650-6655.

Skripuletz, T., Bussmann, J.-H., Gudi, V., Koutsoudaki, P. N., Pul, R., Moharregh-Khiabani, D., Lindner, M., and Stangel, M. (2010). Cerebellar cortical demyelination in the murine cuprizone model. Brain pathology, 20(2):301-312.

Skripuletz, T., Gudi, V., Hackstette, D., and Stangel, M. (2011). De-and remyelination in the cns white and grey matter induced by cuprizone: the old, the new, and the unexpected. Histol Histopathol, 26(12):1585-1597.

Smith, S. M. and Nichols, T. E. (2009). Threshold-free cluster enhancement: Addressing problems of smoothing, threshold dependence and localisation in cluster inference. NeuroImage, 44(1):83 - 98.

Song, S.-K., Yoshino, J., Le, T. Q., Lin, S.-J., Sun, S.-W., Cross, A. H., and Armstrong, R. C. (2005). Demyelination increases radial diffusivity in corpus callosum of mouse brain. NeuroImage, 26(1):132 140.

Stuber, C., Morawski, M., Schäfer, A., Labadie, C., Wähnert, M., Leuze, C., Streicher, M., Barapatre, N., Reimann, K., Geyer, S., Spemann, D., and Turner, R. (2014). Myelin and iron concentration in the 
human brain: A quantitative study of MRI contrast. NeuroImage, 93, Part 1(0):95 - 106.

Sun, S.-W., Liang, H.-F., Trinkaus, K., Cross, A. H., Armstrong, R. C., and Song, S.-K. (2006). Noninvasive detection of cuprizone induced axonal damage and demyelination in the mouse corpus callosum. Magnetic Resonance in Medicine, 55(2):302-308.

Tagge, I., O'Connor, A., Chaudhary, P., Pollaro, J., Berlow, Y., Chalupsky, M., Bourdette, D., Woltjer, R., Johnson, M., and Rooney, W. (2016). Spatio-temporal patterns of demyelination and remyelination in the cuprizone mouse model. PLOS ONE, 11(4):1-24.

Thiessen, J. D., Zhang, Y., Zhang, H., Wang, L., Buist, R., Del Bigio, M. R., Kong, J., Li, X.-M., and Martin, M. (2013). Quantitative MRI and ultrastructural examination of the cuprizone mouse model of demyelination. NMR in Biomedicine, 26(11):1562-1581.

Torkildsen, Ø., Brunborg, L. A., Myhr, K.-M., and B $\varnothing$, L. (2008). The cuprizone model for demyelination. Acta Neurologica Scandinavica, 117:72-76.

Torkildsen, Ø., Brunborg, L. A., Thorsen, F., Mørk, S. J., Stangel, M., Myhr, K.-M., and Bø, L. (2009). Effects of dietary intervention on MRI activity, de- and remyelination in the cuprizone model for demyelination. Experimental Neurology, 215(1):160 - 166.

Turati, L., Moscatelli, M., Mastropietro, A., Dowell, N. G., Zucca, I., Erbetta, A., Cordiglieri, C., Brenna, G., Bianchi, B., Mantegazza, R., Cercignani, M., Baggi, F., and Minati, L. (2014). In vivo quantitative magnetization transfer imaging correlates with histology during de- and remyelination in cuprizone-treated mice. NMR in Biomedicine.

Tustison, N. J., Avants, B. B., Cook, P. A., Zheng, Y., Egan, A., Yushkevich, P. A., and Gee, J. C. (2010). N4itk: Improved $\mathrm{n} 3$ bias correction. IEEE Transactions on Medical Imaging, 29(6):1310-1320.

van Eede, M. C., Scholz, J., Chakravarty, M. M., Henkelman, R. M., and Lerch, J. P. (2013). Mapping registration sensitivity in MR mouse brain images. NeuroImage, 82(0):226 - 236.

Varma, G., Girard, O., Prevost, V., Grant, A., Duhamel, G., and Alsop, D. (2015). Interpretation of magnetization transfer from inhomogeneously broadened lines (ihMT) in tissues as a dipolar order effect within motion restricted molecules in tissues as a dipolar order effect within motion restricted molecules. Journal of Magnetic Resonance, 260:67 - 76.

West, M. J., Slomianka, L., and Gundersen, H. J. G. (1991). Unbiased stereological estimation of the total number of neurons in the subdivisions of the rat hippocampus using the optical fractionator. The Anatomical Record, 231(4):482-497.

Wheeler-Kingshott, C. A. and Cercignani, M. (2009). About "axial" and "radial" diffusivities. Magnetic Resonance in Medicine, 61(5):1255-1260.

Winkler, A. M., Ridgway, G. R., Webster, M. A., Smith, S. M., and Nichols, T. E. (2014). Permutation inference for the general linear model. NeuroImage, 92(0):381 - 397.

Wood, T. C. (2015). Improved formulas for the two optimum VFA flip-angles. Magnetic Resonance in Medicine, 74(1):1-3.

Wu, Q.-Z., Yang, Q., Cate, H. S., Kemper, D., Binder, M., Wang, H.-X., Fang, K., Quick, M. J., Marriott, M., Kilpatrick, T. J., and Egan, G. F. (2008). MRI identification of the rostral-caudal pattern of pathology within the corpus callosum in the cuprizone mouse model. Journal of Magnetic Resonance Imaging, 27(3):446-453.

Xie, M., Tobin, J. E., Budde, M. D., Chen, C.-I., Trinkaus, K., Cross, A. H., McDaniel, D. P., Song, S.-K., and Armstrong, R. C. (2010). Rostrocaudal analysis of corpus callosum demyelination and axon damage across disease stages refines diffusion tensor imaging correlations with pathological features. Journal of Neuropathology \& Experimental Neurology, 69(7):704-716.

Yarnykh, V. L. (2007). Actual flip-angle imaging in the pulsed steady state: A method for rapid threedimensional mapping of the transmitted radiofrequency field. Magnetic Resonance in Medicine, 57(1):192-200.

Yarnykh, V. L. (2010). Optimal radiofrequency and gradient spoiling for improved accuracy of T1 and B1 measurements using fast steady-state techniques. Magnetic Resonance in Medicine, 63(6):1610-1626.

Yarnykh, V. L. and Yuan, C. (2004). Cross-relaxation imaging reveals detailed anatomy of white matter fiber tracts in the human brain. NeuroImage, 23(1):409 - 424.

Zhang, J., Jones, M. V., McMahon, M. T., Mori, S., and Calabresi, P. A. (2012). In vivo and ex vivo diffusion tensor imaging of cuprizone-induced demyelination in the mouse corpus callosum. Magnetic Resonance in Medicine, 67(3):750-759.

Zhang, J., Kolind, S. H., Laule, C., and MacKay, A. L. (2014). Comparison of myelin water fraction from 
675 multiecho T2 decay curve and steady-state methods. Magnetic Resonance in Medicine.

676 Zhu, C., Byrd, R. H., Lu, P., and Nocedal, J. (1997). Algorithm 778: L-bfgs-b: Fortran subroutines for 677 large-scale bound-constrained optimization. ACM Trans. Math. Softw., 23(4):550-560. 